\title{
Ice-shelf buttressing and the stability of marine ice sheets
}

\author{
G. H. Gudmundsson \\ British Antarctic Survey, High Cross, Madingley Rd., Cambridge, CB3 0ET, UK \\ Correspondence to: G. H. Gudmundsson (ghg@bas.ac.uk) \\ Received: 31 July 2012 - Published in The Cryosphere Discuss.: 18 September 2012 \\ Revised: 13 March 2013 - Accepted: 15 March 2013 - Published: 4 April 2013
}

\begin{abstract}
Ice-shelf buttressing and the stability of marinetype ice sheets are investigated numerically. Buttressing effects are analysed for a situation where a stable grounding line is located on a bed sloping upwards in the direction of flow. Such grounding-line positions are known to be unconditionally unstable in the absence of transverse flow variations. It is shown that ice-shelf buttressing can restore stability under these conditions. Ice flux at the grounding line is, in general, not a monotonically increasing function of ice thickness. This, possibly at first somewhat counterintuitive result, is found to be fully consistent with recent theoretical work. Grounding lines on retrograde slopes are conditionally stable, and the stability regime is a non-trivial function of bed and ice-shelf geometry. The stability of grounding lines cannot be assessed from considerations of local bed slope only.
\end{abstract}

\section{Introduction}

A marine-type ice sheet is an ice sheet that rests on a bed located below sea level. Today the prime example of such an ice sheet is the West Antarctic ice sheet (WAIS). In addition to being a marine-type ice sheet, WAIS is mostly located on a retrograde bed, i.e. a bed that generally slopes towards the centre of the ice sheet. It has been argued that marine ice sheets resting on retrograde beds are inherently unstable and subject to possible large-scale disintegration (e.g. Weertman, 1974; Mercer, 1978).

One of the main motivations for studying the stability regime of marine-type ice sheets is the potential for abrupt changes in global sea level. Once perturbed from a steadystate position, an unstable marine-type ice sheet will continue to either advance or retreat, without the need of any additional external forcing, until a new stable steady state is found. A large-scale unstable retreat of WAIS could give rise to several meters of global sea level change within a comparatively short period of time (exact time scale currently unknown, but likely to be on the order of 100 to $1000 \mathrm{yr}$ ).

After decades of work on the marine-ice sheet instability (MISI) hypothesis, and a number of contradictory published findings and statements (e.g. Weertman, 1974; Thomas and Bentley, 1978; Hindmarsh, 1993, 1996; Wilchinsky, 2001, 2009; Schoof, 2007a,b, 2011; Robison et al., 2010) it is now generally accepted that grounding lines of marine ice sheets located on retrograde beds are unconditionally unstable, provided that the flow field does not vary in transverse direction. In the more general geometrical setting where flow and stress fields can vary in both horizontal directions, marine-type ice sheets resting on retrograde slopes are conditionally unstable (Gudmundsson et al., 2012).

Ice-shelf buttressing has been investigated in a number of papers (Dupont and Alley, 2005; Goldberg et al., 2009; Katz and Worster, 2010; Gagliardini et al., 2010). With few exceptions (Goldberg et al., 2009; Katz and Worster, 2010) most studies have analysed buttressing effects using flowline models. It will be argued below that ice-shelf buttressing is inherently a three-dimensional effect and that being able to accurately account for variations in stresses in both horizontal dimensions is an essential prerequisite for any studies of this effect on grounding line stability. Although flow-line studies are, in this context, arguably of somewhat limited use, they have nevertheless convincingly illustrated the potential of ice-shelf buttressing in affecting the dynamics of marinetype ice sheets.

This work is an extension, and can be regarded as an accompanying paper, of the recent study by Gudmundsson et al. (2012). In Gudmundsson et al. (2012) specific numerical examples of stable grounding lines on retrograde slopes are provided. Here the main focus is on the stress balance at the grounding line and on the role of ice-shelf buttressing in 
restoring stability. The analysis is done for the examples presented in Gudmundsson et al. (2012). In the following it will be assumed that the reader is able to consult that paper for a more detailed description of some of the numerical aspects of this work.

The paper is organised as follows: First the concept of iceshelf buttressing is explained and a number of parameters used to quantify and describe the effects of ice-shelf buttressing on grounded ice sheets are introduced. This is followed by a description of the particular ice-flow problem considered here, listing of all modelling parameters, and a brief description of the numerical model. The following main bulk of the paper focuses on an analysis of the stress regime along the grounding line, and on the role of ice-shelf buttressing in affecting the state of stress, ice flux, and the stability regime of marine ice sheets.

\section{Ice-shelf buttressing}

Ice-shelf buttressing can be defined as the mechanical effect of an ice shelf on the state of stress at the grounding line. Along the grounding line, the grounded and the floating parts of the ice are in direct contact. The state of stress at the grounding line can, in general, be expected to be affected by the presence of the floating ice shelf. If the ice shelf were to be removed, the (new) calving front at the grounding line would find itself in direct contact with the ocean. The resulting change in the stress at the grounding line can be taken as a measure of ice-shelf buttressing.

As explained below, following the removal of a laterally unconfined ice shelf, and in the absence of any variations in the transverse direction, the net change in (vertically averaged) grounding-line stress is zero. Hence, in one horizontal dimension (1HD) unconfined ice shelves do not give rise to any buttressing. This is the reason why buttressing is inherently a process that must be studied in two horizontal dimensions (2HD) using, at the minimum, models that can resolve stress in both horizontal directions.

Following Morland (1987) and MacAyeal (1989), the horizontal balance of stresses in an ice stream and an ice shelf can be written in a vertically integrated form as

$\nabla_{h}^{T} \cdot(h \boldsymbol{T})-\boldsymbol{t}_{b h}=\rho g h \nabla_{h} s$,

where

$\boldsymbol{T}=\left(\begin{array}{cc}2 \tau_{x x}+\tau_{y y} & \tau_{x y} \\ \tau_{x y} & 2 \tau_{y y}+\tau_{x x}\end{array}\right)$

with

$\nabla_{h}^{T}=\left(\partial_{x}, \partial_{y}\right)$.

In the above equation, $\tau_{i j}$ are the components of the deviatoric stress tensor, $s$ is the surface topography, $h$ is the ice thickness, $\rho$ is the ice density, $g$ is the gravitational acceleration, and $t_{b h}$ is the horizontal part of the bed-tangential basal traction $\boldsymbol{t}_{b}$ where

$\boldsymbol{t}_{b}=\boldsymbol{\sigma} \hat{\boldsymbol{n}}-\left(\hat{\boldsymbol{n}}^{T} \cdot \boldsymbol{\sigma} \hat{\boldsymbol{n}}\right) \hat{\boldsymbol{n}}$,

with $\hat{\boldsymbol{n}}$ being a unit normal vector to the bed pointing into the ice. When used as a subscript, $h$ is a mnemonic for 'horizontal'.

The key assumptions leading to Eq. (1) are (1) $u_{\mathrm{b}} / u_{\mathrm{d}} \gg$ 1 where $u_{\mathrm{b}}$ is the basal sliding velocity and $u_{\mathrm{d}}$ the internal deformation velocity, and (2) the analysis is limited to large spatial variations compared to mean ice thickness (e.g. Baral and Hutter, 2001; Gudmundsson, 2008).

For a floating calving front the stress boundary condition is

$\boldsymbol{T} \hat{\boldsymbol{n}}_{c}=\frac{1}{2} \varrho g h \hat{\boldsymbol{n}}_{c}$,

where

$\varrho=\rho\left(1-\rho / \rho_{w}\right)$,

with $\rho_{w}$ being the ocean density. In Eq. (5) the unit vector $\hat{\boldsymbol{n}}_{c}$ points horizontally outwards away from the calving front.

For the following discussion, it is convenient to define

$N=\hat{\boldsymbol{n}}_{g l}^{T} \cdot\left(\boldsymbol{T} \hat{\boldsymbol{n}}_{g l}\right)$,

and

$T=\hat{\boldsymbol{m}}^{T} \cdot\left(\boldsymbol{T} \hat{\boldsymbol{n}}_{g l}\right)$,

where

$\hat{\boldsymbol{n}}_{g l}^{T}=\left(n_{x}, n_{y}, 0\right)^{T}$,

is a unit normal to the grounding line, and

$\hat{\boldsymbol{m}}^{T}=\left(-n_{y}, n_{x}, 0\right)^{T}$.

The vector $\hat{\boldsymbol{m}}$ is both normal to $\hat{\boldsymbol{n}}_{g l}$ and tangential to the grounding line.

If the ice shelf were momentarily to be removed without affecting the position of the grounding line, the grounding line and the calving front would coincide and $\hat{\boldsymbol{n}}_{g l}=\hat{\boldsymbol{n}}_{c}$, i.e. the normal to the grounding line $\left(\hat{\boldsymbol{n}}_{g l}\right)$ and the normal to the calving front $\left(\hat{\boldsymbol{n}}_{c}\right)$ would be identical. Hence, in the absence of an ice shelf

$N=\frac{1}{2} \varrho g h$,

and

$T=0$,

where the boundary condition (5) and the definitions of $N$ and $T$ given by (6) and (7) have been used. In general $T$ and 
$N$ will not be given by Eqs. (8) and (9) because the stresses at the grounding line will be affected to some degree by the presence of the ice shelf. How strongly $N$ and $T$ are affected by the ice shelf can be taken as a measure of the importance of buttressing. A convenient measure of the degree of iceshelf buttressing are the numbers

$K_{N}=\frac{N_{0}-N}{N_{0}}$,

and

$K_{T}=\frac{T}{N_{0}}$,

where $N_{0}$ is defined to be the ice-shelf-free value of $N$, i.e. the value of $N$ in the absence of an ice shelf, hence

$N_{0}=\frac{1}{2} \varrho g h$.

The numbers $K_{M}$ and $K_{T}$ will here be referred to as the normal and tangential buttressing numbers, respectively.

The normal buttressing number $K_{N}$ is the normalised difference between (a) the (vertically integrated) ocean pressure that would act in horizontal direction along the grounding line in the absence of the ice shelf, and (b) the actual (vertically integrated) normal stress. If $K_{N}>0$, then normal stresses at the grounding line are smaller than they otherwise would be in the absence of an ice shelf, and the ice shelf can be considered to be restricting the ice motion at the grounding line more than the ocean would otherwise do. For $K_{N}<0$, the tensile stresses at the grounding line are larger than they would be with the ice shelf removed, and the ice shelf is 'pulling' at the grounding line.

The degree of buttressing can also be quantified by introducing a (normal) buttressing ratio $\Theta_{N}$ defined as

$\Theta_{N}=\frac{N}{N_{0}}$,

and a tangential buttressing ratio as

$\Theta_{T}=\frac{T}{N_{0}}$.

\subsection{Ice-shelf buttressing in one horizontal dimension}

Although a simple point, and one that has been raised repeatedly in a number of papers previously (e.g. MacAyeal and Barcilon, 1988; Schoof, 2007a) it is worth stressing that in one horizontal dimension (1HD) ice-shelf buttressing is absent. This can be seen for example by considering in 1HD first the boundary condition (5), which reads

$\tau_{x x}=\frac{1}{4} \varrho g h$,

and subsequently the stress-equilibrium equation (1), which, when expressed at the ocean side of the grounding line (i.e. limit taken from the ocean side) where basal shear stress disappears and $\partial_{x} s=\left(1-\rho / \rho_{w}\right) \partial_{x} h$, reduces, after an integration with respect to $x$, to

$\tau_{x x}=\frac{1}{4} g \varrho h$

where Eq. (15) has been used to show that the integration constant is equal to zero. Hence, in 1HD, the stress conditions at the calving front and at the grounding line are identical. If the ice-shelf were to be removed, the stress condition at the grounding line would no longer be given by Eq. (16) but by Eq. (15), but as this new condition is identical to the previous one the state of stress at the grounding line is not affected by the ice-shelf's disappearance. In 1HD ice-shelves are thus "passive" and do not affect the stresses at or upstream from the grounding line. It also follows that ice-shelf melting, iceshelf calving or any other changes in the conditions of the ice shelf, have no effect on the position or the stability of the grounding line. Furthermore, following an ice-shelf collapse, ice flux at the grounding line remains unchanged.

In 1HD the ice-shelf buttressing numbers $K_{N}$ and $K_{T}$ are both identically equal to zero. The normal buttressing ratio $\Theta_{N}$ is equal to unity, and the tangential buttressing ratio $\Theta_{T}$ is equal to zero.

It should be noted that these statements above about buttressing in 1HD, and unconfined ice shelves being passive, follow from and can be considered to be properties of the system (Eq. 1). This system represents a reduced version of the full Stokes system. In the more general case where the full Stokes system needs to be considered these statements no longer hold. A particular example is bending stresses, i.e. vertical variations in horizontal stresses, which are not accounted for in Eq. (1). In 1HD such bending stresses can, for example, be set up by tides acting on an ice shelf. Numerical modelling of ice-shelf/ice-stream interaction in 1HD using the full Stokes system (Gudmundsson, 2011) shows tidal stresses to have the potential to significantly affect horizontal motion over large distances upstream from the grounding line (large compared to mean ice thickness). Removing the ice shelf would affect the bending stresses at the grounding line. Hence, in this situation an unconfined ice shelf is not passive.

It should also be noted that unconfined ice shelves, in the general 2HD setting, can affect the stresses at the grounding line. This can, for example, happen for a straight grounding line and unconfined ice shelf, if the calving front has as complex shape. In that case the stress distribution within the ice shelf can be expected to be complex and to no longer vary in one spatial direction only. The stresses at the grounding line will then, in general, also change with distance and the pointwise equality between normal stresses at the grounding line and the ocean pressure no longer holds. 


\subsection{Ice-shelf buttressing in two-horizontal dimensions as a possible stabilising factor}

As explained by Schoof (2007a, 2012), if ice flux is an increasing function of ice thickness at the grounding line, steady-state grounding-line position of marine ice sheets on retrograde slopes are unstable. Provided some simplifying assumptions - similar to but somewhat stronger than to those leading to Eq. (1) - are made (see Schoof, 2007a), steadystate ice flux $(q)$ at the grounding line can be written as

$$
\begin{aligned}
q= & \rho\left(\frac{A(\rho g)^{n+1}\left(1-\rho / \rho_{w}\right)^{n}}{4^{n}} C^{-1 / m}\right)^{m /(1+m)} \\
& \Theta_{N}^{n m /(1+m)} h^{(1+m(n+3)) /(1+m)} .
\end{aligned}
$$

Note that Eq. (17) is given in a notation slightly different from the one used in Schoof (2007a), and that $q$ is here in the SI units of $\mathrm{kg} \mathrm{s}^{-1} \mathrm{~m}^{-1}$. The parameters $C$ and $m$ are the basal slipperiness and the stress exponent, respectively, of Weertman's sliding law where

$\boldsymbol{t}_{b}=C^{-1 / m}\left|\boldsymbol{v}_{b}\right|^{1 / m-1} \boldsymbol{v}_{b}$,

with

$\boldsymbol{v}_{b}=\boldsymbol{v}-\left(\hat{\boldsymbol{n}}^{T} \cdot \boldsymbol{v}\right) \hat{\boldsymbol{n}}$

being the basal sliding velocity. $A$ and $m$ are parameters in Glen's flow law

$\dot{\epsilon}_{i j}=A \tau^{n-1} \tau_{i j}$,

where $\tau$ is the second invariant of the deviatoric stress tensor

$\tau=\sqrt{\tau_{p q} \tau_{p q} / 2}$,

and were $\dot{\epsilon}_{i j}$ are the strain rates. The parameter $\Theta_{N}$ is the (normal) buttressing ratio defined above by Eq. (13), and $h$, $\rho$, and $\rho_{w}$ are the ice thickness and the ice and ocean densities, respectively.

The validity of Eq. (17) in general 2HD setting still remains to be assessed. A simple derivation of the flux relationship in 1HD can be found in (Hindmarsh, 2012), showing that the equation is based on steady-state considerations and the assumption that horizontal stress gradients are small in comparison to basal drag. In $2 \mathrm{HD}$, following Schoof (2007a, 2012), further assumptions are that the curvature of the grounding line is not too large, and the ratio between tangential and normal buttressing small, i.e. $T / N \ll 1$.

Equation (17) predicts ice flux to be an increasing function of both ice thickness $h$ and the normal buttressing ratio $\Theta_{N}$. The normal buttressing ratio $\Theta_{N}$ can in turn be expected to depend on ice thickness, but in Schoof's theory the dependency of $\Theta_{N}$ on $h$ is unspecified and needs to be determined numerically. Within the context of Eq. (17) grounding lines on retrograde slopes are unstable provided $\Theta_{N}$ either increases with $h$, or decreases sufficiently slowly with $h$, for $q$ to be an increasing function of $h$.

In 1HD the normal buttressing ratio $\Theta_{N}$ is, as mentioned above, always equal to unity, and ice flux is therefore always an increasing function of thickness. Grounding lines on retrograde slopes in 1HD are therefore unstable. In 2HD no such specific and precise statements about grounding-line stability can be made on the basis of Eq. (17). What Eq. (17) however clearly illustrates is the importance of ice-shelf buttressing for the stability of grounding lines. In particular, the equation shows that ice-shelf buttressing can potentially act as a stabilising factor for grounding lines.

\section{Problem definition}

The bed geometry is motivated by the synthetic bed shape used in the flow-line studies by Schoof (2007a) and Pattyn et al. (2012). Here, this bed profile has been extended by introducing additional variations in transverse direction. The resulting bed is that of a longitudinal channel incised into a slowly undulating plane, with an overall downward slope in the $x$ direction (see Fig. 1 in Gudmundsson et al., 2012). The only parameter describing the bed that is varied in the examples provided below is the half-width of the channel.

Although the bed geometry and model parameters are identical to those used in Gudmundsson et al. (2012), the equation defining the bed and all model parameters are listed below for convenience.

The bed is defined as

$B(x, y)=B_{x}(x)+B_{y}(y)$,

where

$$
\begin{aligned}
B_{x}(x)=B_{0} & -2184.8\left(x / 750 \times 10^{3}\right)^{2} \\
& +1031.72\left(x / 750 \times 10^{3}\right)^{4} \\
& -151.72\left(x / 750 \times 10^{3}\right)^{6},
\end{aligned}
$$

and

$B_{y}(y)=\frac{d_{c}}{1+e^{-2\left(y-w_{c}\right) / f_{c}}}+\frac{d_{c}}{1+e^{2\left(y+w_{c}\right) / f_{c}}}$.

$B(x, y)$ stands for the topography of the ocean floor, and the units are meters. All model parameters are listed in Table 1.

Note that the slope of the bed in $x$ direction is independent of $y$, i.e. $\partial_{y x}^{2} B(x, y)=0$, and that the bed slope in $x$ direction is zero at both $x=0, x=x_{a}$ and $x=x_{b}$ where $x_{a}=973.7 \mathrm{~km}$ and $x_{b}=1265.7 \mathrm{~km}$. The region $x_{a}<x<x_{b}$, independently of the value of $y$, is an area of retrograde bed slope, where the bed slopes upwards with increasing $x$, i.e.

$\partial_{x} B(x, y)>0$ for $x_{a}<x<x_{b}$.

The model domain stretches from 0 to $1800 \mathrm{~km}$ in $x$ direction, and from -120 to $120 \mathrm{~km}$ in $y$ direction. 
Table 1. Model parameters. The parameters $B_{0}, f_{c}$ and $d$ are geometrical parameters that affect the shape of the bedrock (see Eqs. 22 to 24). $A$ and $n$ are the rate factor and the stress exponent of Glen's flow law, respectively, $C$ and $m$ are the basal slipperiness and the stress exponent of Weertman's sliding law, and $\rho$ and $\rho_{w}$ are the specific densities of ice and ocean. The variable $a$ is the surface mass balance in the units of ice equivalent. The number of days in a year is 365.25 .

\begin{tabular}{lll}
\hline Parameter & Value & Units \\
\hline$B_{0}$ & 300 & $\mathrm{~m}$ \\
$f_{c}$ & 5000 & $\mathrm{~m}$ \\
$d_{c}$ & 1000 & $\mathrm{~m}$ \\
$A$ & $10^{-24}$ & $\mathrm{~s}^{-1} \mathrm{~Pa}^{-3}$ \\
$n$ & 3 & \\
$C$ & $2.256 \times 10^{-21}$ & $\mathrm{~m} \mathrm{~s}^{-1} \mathrm{~Pa}^{-3}$ \\
$m$ & 3 & \\
$\rho$ & 900 & $\mathrm{~kg} \mathrm{~m}^{-3}$ \\
$\rho_{w}$ & 1000 & $\mathrm{~kg} \mathrm{~m}^{-3}$ \\
$a$ & 0.3 & $\mathrm{ma}^{-1}$ \\
\hline
\end{tabular}

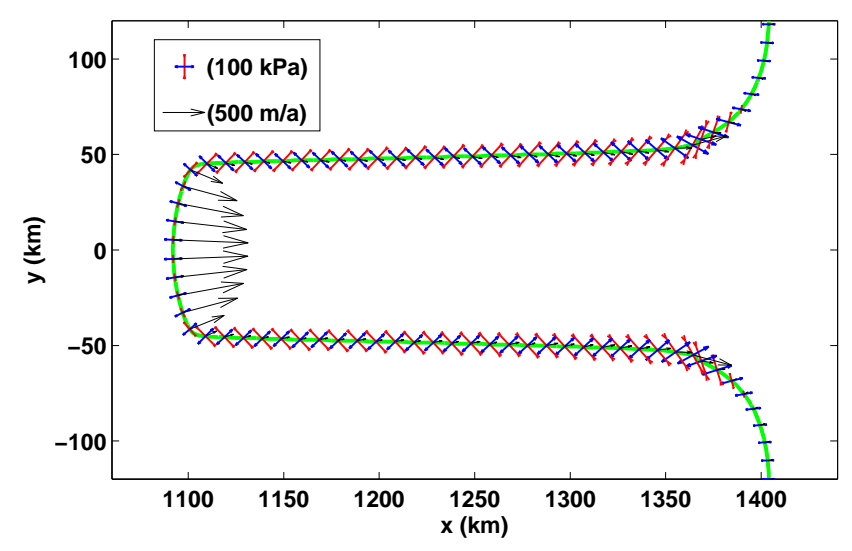

Fig. 1. Principal deviatoric stresses and horizontal velocities along the grounding line for a $100 \mathrm{~km}$-wide channel $\left(w_{c}=50 \mathrm{~km}\right)$. All other model parameters are listed in Table 1. Only a part of the model domain is shown. The overall flow direction is from left to right. Grounding line is shown in green. Ice to the left of the grounding line is grounded (ice stream) and ice to the right of the grounding line is afloat (ice shelf). Tensile stresses are shown in blue and compressive stresses in red. Bed slopes are retrograde for $973.7 \mathrm{~km}<x<1265.7 \mathrm{~km}$.

For $x=0$, both horizontal velocity components are set to zero, i.e. $u(0, y)=v(0, y)=0$, and along the sides where $y= \pm 120 \mathrm{~km}$, the $y$ velocity component is set to zero, i.e. $v(x, \pm 120 \mathrm{~km})=0$. The ice velocities are, hence, set to zero in both $x$ and $y$ directions along the upper limit of the model domain $(x=0)$, and the ice is allowed to slip freely along the left and right sides $(y= \pm 120 \mathrm{~km})$. Shear stresses along the left and the right hand sides are set to zero. The calving front is situated at $x=1800 \mathrm{~km}$ and boundary condition applied along the calving front is the (vertically integrated) ocean pressure (see Eq. 5).

\section{Numerical model}

The numerical model (referred to as "Úa") has been repeatedly used in the past to solve problems involving grounding-line migration, and results from the model were submitted to both the MISMIP and the MISMIP3D modelintercomparison exercises (Pattyn et al., 2012, 2013).

The numerical model solves the equations

$$
\nabla_{h}^{T} \cdot(h \boldsymbol{T})-\boldsymbol{t}_{b h}=\rho g h \nabla_{h} s,
$$

and

$\partial_{t} h+\partial_{x}(u h)+\partial_{y}(v h)=a$,

together with corresponding boundary conditions, using the method of finite elements. In the equation above, $a$ is the mass balance. The time evolution is calculated in a fully coupled manner where both Eqs. (1) and (26) are solved simultaneously and implicitly, with the changes in $u, v$ and $h$ as unknowns, using a third-order Taylor-Galerkin method. At each time step the resulting non-linear system is solved using the Newton-Raphson method.

\section{Results}

Ice flow over the bed defined by Eq. (22) using the model parameters listed in Table 1 was calculated numerically until the mean rate of surface elevation change was less than $0.001 \mathrm{ma}^{-1}$, in which case a steady state was considered to have been reached. As described in Gudmundsson et al. (2012), a number of steady-state examples were found with grounding lines located on the retrograde section of the bed. During the course of the model runs, the grounding lines slowly migrated towards these retrograde sections of the bed, and the possibility that final steady-state grounding-line positions are unstable can be discounted.

In the following I will start by presenting a few examples of calculated deviatoric stresses and buttressing numbers along the grounding line for a one particular geometrical setup, before moving to the main conclusions of the paper about the relationship between ice flux and ice thickness.

\subsection{Examples of variations in deviatoric stresses and ice-shelf buttressing along the grounding line}

Figure 1 shows the deviatoric stresses and velocities along the grounding line. Here the half-width $\left(w_{c}\right)$ of the incised channel was set to $50 \mathrm{~km}$, with all other model parameters taken from Table 1 . The figure shows that by far the largest ice velocities along the grounding line are found within the main ice channel, i.e. between $-50 \mathrm{~km}<y<$ $50 \mathrm{~km}$ and for $x \approx 1100 \mathrm{~km}$. As pointed out above the bed slope is retrograde for $x_{a}<x<x_{b}$, where $x_{a}=973.7 \mathrm{~km}$ and $x_{b}=1265.7 \mathrm{~km}$. The grounding line shown in Fig. 1 is, hence, in parts located on a retrograde sloping bed and, in 


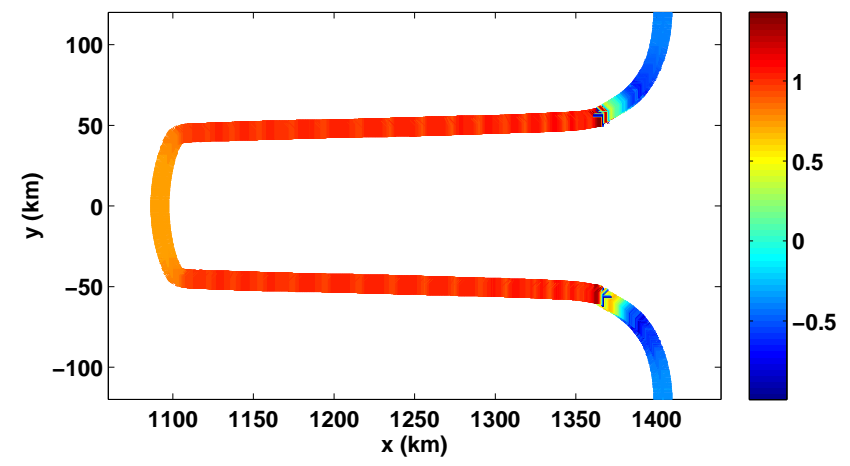

Fig. 2. The normal buttressing number $K_{N}$ (defined by Eq. 10). The value of $K_{N}$ is shown along the grounding line. The width of the coloured line in the plot is chosen for clarity and does not reflect the actual width of the grounding line. All model parameters are the same as for Fig. 1.

particular, the bed slope is retrograde over the section of the bed with highest velocities and greatest ice flux.

In $1 \mathrm{HD}$, deviatoric stresses at the grounding line are always tensile in direction normal to the grounding line, and zero in tangential direction. Figure 1 depicts a somewhat more complicated stress regime. Along the section of the grounding line furthest upstream (i.e. for $-50 \mathrm{~km}<y<$ $50 \mathrm{~km}$ and for $x \approx 1100 \mathrm{~km}$ ) deviatoric stresses are tensile in normal direction and compressive tangential to the grounding line. The margins of the confined ice shelf are, on the other hand, subjected to shear, and in terms of the magnitude of the principal deviatoric stress components, these are the sections of the grounding line subjected to the largest deviatoric stress.

The degree of buttressing is shown in Figs. 2 and 3 displaying the buttressing numbers $K_{N}$ and $K_{T}$, respectively, as defined by Eqs. (10) and (11). As explained above, the normal buttressing number is always equal to zero in 1HD.

In the particular case illustrated in Fig. 2, the normal buttressing number $K_{N}$ varies between about -0.7 to 1.5 . A value of 1.5 implies that the difference between the normal component of the normal stress vector at the grounding line with and without an ice shelf is 1.5 times larger than the stress in the ice-shelf-free case. Measured in this way, the state of stress along the grounding line is, hence, significantly affected by the presence of the ice shelf. Where the medial line crosses the grounding line, $K_{N}$ is around 0.6 implying that the normal stress is only $60 \%$ of the corresponding iceshelf-free value. In this region the ice shelf restricts ice motion across the grounding line and the spreading rate at the grounding line would be significantly larger with the ice shelf removed. On the other hand, further downstream the normal buttressing values become negative (see Fig. 2) showing that in these regions the ice shelf "pulls" the ice at the grounding line forward.

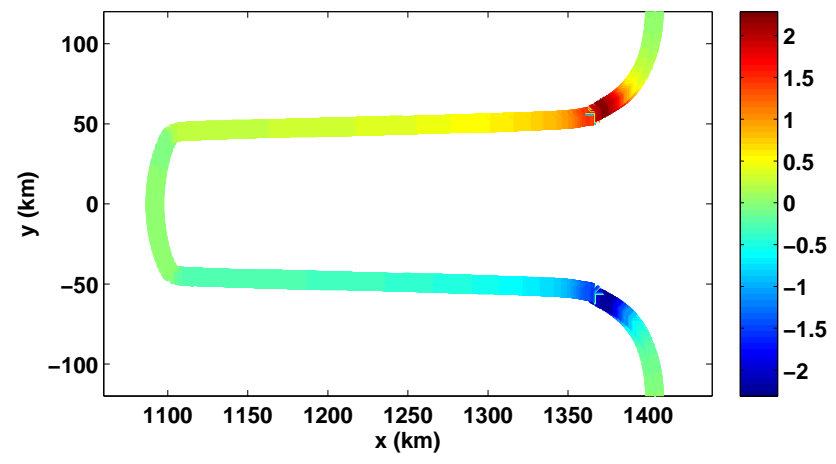

Fig. 3. The tangential buttressing number $K_{T}$ (defined by Eq. 11). All model parameters are the same as for Fig. 1.

The tangential buttressing number, $K_{T}$, shown in Fig. 3 varies between about -2 and 2 but is mostly in magnitude smaller than about 0.5. As Eq. (11) shows, the tangential buttressing number is normalised by the vertically-averaged horizontal pressure of the ocean that would act in the horizontal plane along the grounding line where the ice shelf to be removed. In $1 \mathrm{HD}, K_{T}$ is always equal to zero.

\subsection{Ice flux along the grounding line}

Model intercomparison studies of grounding-line motion have demonstrated the need for high spatial resolution, on the order of one ice thickness, in the vicinity of the grounding line (Pattyn et al., 2012). For practical reasons, numerical modelling of large-scale ice sheets using structured grids with that degree of spatial resolution is not feasible. It has been suggested (e.g. Pollard and DeConto, 2012) that a possible way of avoiding the requirement for such a high spatial resolution is to prescribe, rather than to calculate, ice flux at the grounding line using Eq. (17). Such a modelling approach depends on both (1) the models ability to accurately calculate the normal buttressing ratio $\left(\Theta_{N}\right)$ for relatively coarse grid resolution, and (2) the correctness of Eq. (17) in a general setting where some of the assumptions behind that equation, such as steady-state conditions, may not be strictly fulfilled.

The numerical model, Úa, employed here does not rely on a flux parametrisation of this type. It uses unstructured grids with an automated mesh refinement around the grounding line, and solves equations commonly used in glaciology to describe the flow of ice streams and ice shelves. The question arises if ice fluxes calculated with Úa agree with the fluxes predicted by Eq. (17).

The normal buttressing ratio $\Theta_{N}$ given by the numerical model was inserted into Eq. (17) and the ice flux as predicted by Eq. (17) calculated. Figure 4 shows both the ice flux given by the numerical model (blue line), and the ice flux based on Eq. (17) (red). As Fig. 4 shows, the difference between numerically calculated ice fluxes normal to the grounding line and those given by Eq. (17) ranges from about $-20 \%$ to about $+50 \%$. However, where the grounding line crosses 


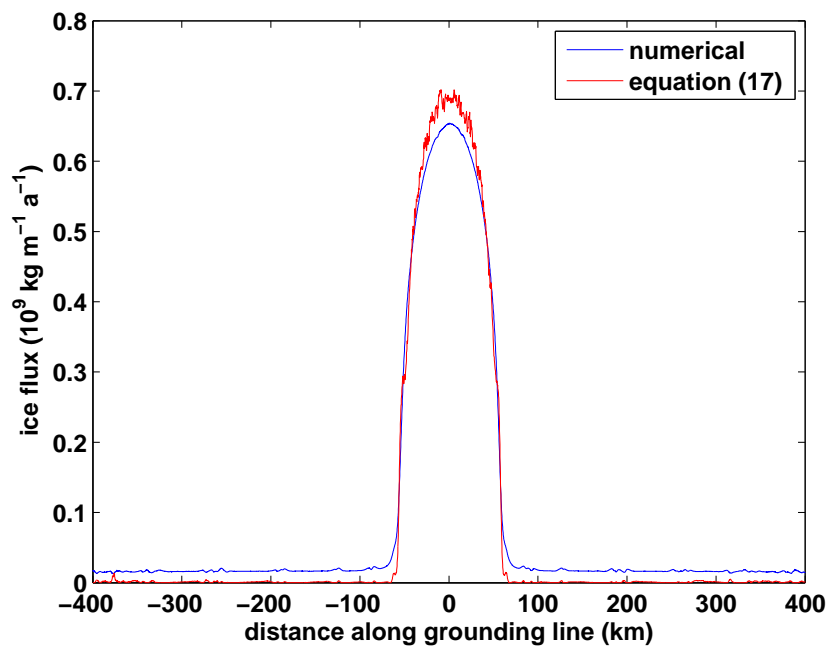

Fig. 4. Modelled ice flux across the grounding line (blue), and ice flux calculated from Eq. (17) (red) from Schoof (2007a). The results shown are for a channel with a half-width $w_{c}=60 \mathrm{~km}$. The $x$ axis shows the distance along the grounding line, with $x=0$ being at the symmetry axis of the model domain where the grounding line crosses the medial line. Despite the grounding line curving somewhat in that region, the range $-60 \mathrm{~km} \leq x \leq 60 \mathrm{~km}$ broadly coincides with the deepest section of the channel. The flux $(q)$ shown is the vertically integrated flux, i.e. $q=\rho h v_{n}$, where $v_{n}$ is the velocity normal to the grounding line.

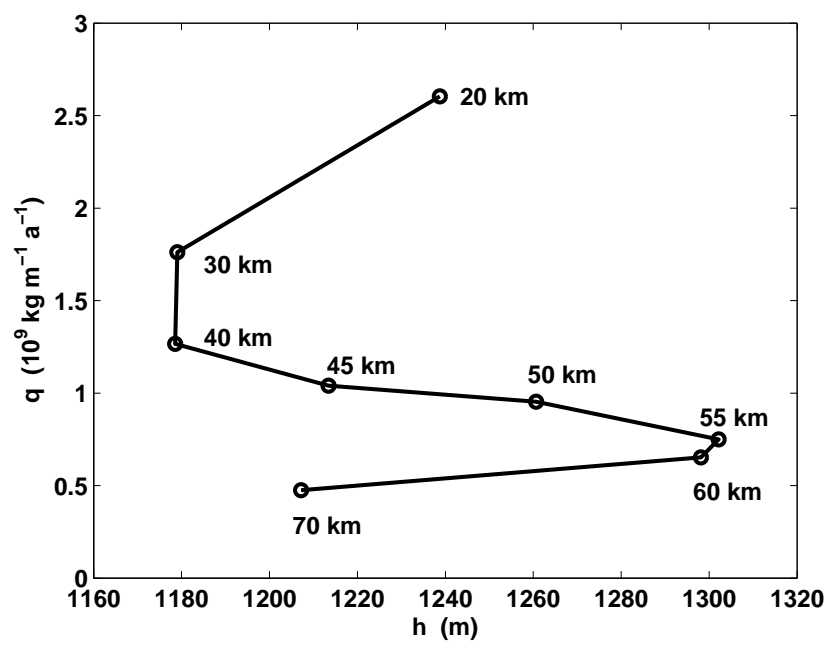

Fig. 5. Ice flux normal to the grounding line as a function of ice thickness. Each data point is calculated for a different model setup with half-widths values, ( $w_{c}$, see Eqs. 22 to 24 ) ranging from 20 to $70 \mathrm{~km}$. In each case the flux values are from the locations where the grounding lines of the respective models cross the medial line $(y=0)$.

the medial line, where $x=0$ in the figure, the difference is only about $6 \%$.

The normal flux was integrated over the horizontal distance along the grounding line giving the total ice flux across

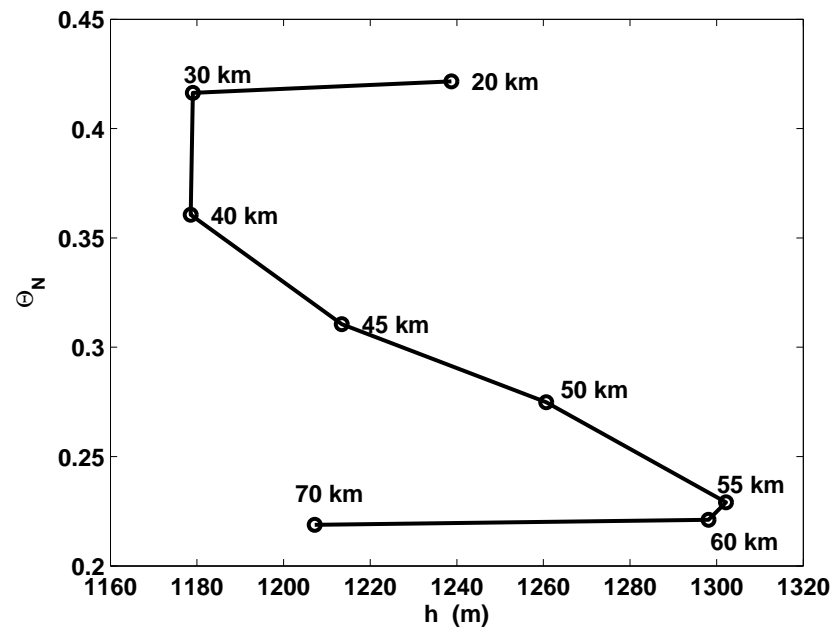

Fig. 6. The normal buttressing ratio $\Theta_{N}$ where the medial line $(y=0)$ crosses the grounding line as a function of ice thickness. As in Fig. 5 each data point is calculated for a different model setup with varying half-widths values, ( $w_{c}$, see Eqs. 22 to 24 ) from 20 to $70 \mathrm{~km}$. The normal buttressing ratio is defined by Eq. (13) and reflects the difference in stress, or more precisely the difference in the normal component of the normal stress vector, at the grounding line in the presence and in the absence of an ice shelf.

the grounding line. Comparison between the total ice flux based on Eq. (17), and ice flux given directly by the numerical model, revealed differences ranging from 13 to $19 \%$ depending on channel width. As Fig. 4 shows, for $w_{c}=60 \mathrm{~km}$ ice fluxes along the ice-shelf margins are underestimated by Eq. (17) and overestimated within the deepest part of the channel. This was also found to be the case for other halfwidths values ranging from $w_{c}=20 \mathrm{~km}$ to $w_{c}=70 \mathrm{~km}$.

Depending on the situation, the differences between ice fluxes obtained directly from the numerical model, or with the help of Eq. (17), may or may not be of importance. Generally, one might expect a difference of less than about $10 \%$ to be quite acceptable. These result can therefore be taken to give an increased confidence in the use of Eq. (17) as a flux parametrisation in large-scale flow models. However, Eq. (17) can only be used to arrive at grounding-line fluxes once the normal buttressing ratio $\Theta_{N}$ has been estimated. Obtaining such estimates, in turn, requires the use of a numerical model. When using a numerical model that does not employ a high resolution grid around the grounding line to calculate the normal buttressing ratio $\left(\Theta_{N}\right)$, the resulting error in calculated fluxes using Eq. (17) could be much larger than suggested by the numbers listed above.

\subsection{Ice flux and buttressing ratios as functions of ice thickness}

As mentioned above, a simple heuristic argument (e.g. Weertman, 1974; Schoof, 2007a) shows that marine ice sheets resting on retrograde slopes are unstable provided ice 
flux at the grounding line is a monotonically increasing function of ice thickness.

Figure 5 shows numerically calculated ice fluxes at the grounding lines for a number of bed geometries as a function of ice thickness. The fluxes are in each case calculated at locations where the grounding lines of individual model setups cross the medial line $(y=0)$. The only model parameter that is varied is the half-width $\left(w_{c}\right)$ of the incised channel. The grounding lines were located on retrograde slopes for $w_{c}=40,45$, and $50 \mathrm{~km}$, and on prograde slopes for $w_{c}=20$, 30, 60 and $70 \mathrm{~km}$. As Fig. 5 shows, calculated ice flux is not a monotonically increasing function of ice thickness. In the instances where the grounding lines are located on retrograde slopes $\left(w_{c}=40\right.$ to $\left.w_{c}=50\right)$ ice flux decreases with increasing thickness. In numerical terms the decrease can be considered to be rather large. While the thickness at the grounding line increases from about 1180 to $1270 \mathrm{~m}$, or about $7 \%$, the ice flux decreases by about $30 \%$ (see Fig. 5). For those models where the grounding lines are located on prograde slopes, flux, on the other hand, increases with increasing thickness. This can be seen following the change in flux from $w_{c}=70 \mathrm{~km}$ to $w_{c}=60 \mathrm{~km}$, and from $w_{c}=30 \mathrm{~km}$ to $w_{c}=20 \mathrm{~km}$.

The normal buttressing ratio, $\Theta_{N}$, is depicted in Fig. 6 in a similar fashion to Fig. 5, i.e. as a function of ice thickness at the $x y$ locations where the grounding lines cross the medial line. Figure 6 shows that buttressing can decrease with increasing thickness. As shown above, at the $x y$ locations of the data points in both Figs. 5 and 6, calculated ice fluxes agree to within a few percent with Eq. (17). Thus, in the context of Eq. (17) where ice flux is considered to be a function of thickness and the normal buttressing ratio, the reason for decreasing ice flux with increasing ice thickness - observed for all models with grounding lines located on retrograde slopes - is the change in the normal buttressing ratio. It follows that ice-shelf buttressing can be said to be directly responsible for the stability of these grounding lines.

\section{Summary and conclusions}

Ice-shelf buttressing can stabilise otherwise unstable grounding-line positions of marine-type ice sheets. It follows that the effects of ice-shelf buttressing need to be accurately accounted for in large-scale models of marine-type ice sheets for such models to have any predictive power. Ice-shelf buttressing is inherently a three-dimensional process requiring calculations of stress balance in both horizontal dimensions. Unless transverse stress variations can be sufficiently well parametrised, the use of flow-line models to study the mechanical effects of ice-shelves on the force balance at the grounding line is problematic.

Due to buttressing effects, ice flux at the grounding line can be a decreasing function of ice thickness. Grounding lines on retrograde bed slopes are then no longer unstable.
Modelled steady-state ice fluxes agree favourably with Eq. (17) based on Schoof (2007a). In some recent numerical models, Eq. (17) is used as an internal flux condition defining ice flux at the grounding line (Pollard and DeConto, 2012). The good agreement found here is encouraging and, provided numerical flow models of this type can give accurate estimates of the normal buttressing ratio, supports this use of Eq. (17). However, it should be noted that Eq. (17) can only be used once buttressing effects have been calculated, which in turn requires the use of a numerical model.

Grounding lines on retrograde slopes are conditionally stable, and the stability regime a non-trivial function of bed and ice-shelf geometry. Except in the limited case where transverse variations in flow field are absent, it appears unlikely that the stability of grounding lines can be judged from simple geometrical considerations alone.

Acknowledgements. This work was supported by NERC grant $\mathrm{Nr}$. NE/H02333X/1. The support by J. Vosper is acknowledged. I thank R. Arthern for a number of very helpful and constructive comments.

Edited by: E. Larour

\section{References}

Baral, D. and Hutter, K.: Asymptotic Theories of Ice Sheets and Ice Shelves, in: Geomorphological Fluid Mechanics, edited by: Balmforth, N. and Provenzale, A., vol. 582 of Lecture Notes in Physics, 227-278, Springer Berlin/Heidelberg, 2001.

Dupont, T. K. and Alley, R. B.: Assessment of the importance of ice-shelf buttressing to ice-sheet flow, Geophys. Res. Lett., 32, L04503, doi:10.1029/2004GL022024, 2005.

Gagliardini, O., Durand, G., Zwinger, T., Hindmarsh, R. C. A., and Meur, E. L.: Coupling of ice-shelf melting and buttressing is a key process in ice-sheets dynamics, Geophys. Res. Lett., 37, L14501, doi:10.1029/2010GL043334, 2010.

Goldberg, D., Holland, D. M., and Schoof, C.: Grounding line movement and ice shelf buttressing in marine ice sheets, J. Geophys. Res., 114, F04026 doi:10.1029/2008JF001227, 2009.

Gudmundsson, G. H.: Analytical solutions for the surface response to small amplitude perturbations in boundary data in the shallow-ice-stream approximation, The Cryosphere, 2, 77-93, doi:10.5194/tc-2-77-2008, 2008.

Gudmundsson, G. H.: Ice-stream response to ocean tides and the form of the basal sliding law, The Cryosphere, 5, 259-270, doi:10.5194/tc-5-259-2011, 2011.

Gudmundsson, G. H., Krug, J., Durand, G., Favier, L., and Gagliardini, O.: The stability of grounding lines on retrograde slopes, The Cryosphere, 6, 1497-1505, doi:10.5194/tc-6-1497-2012, 2012.

Hindmarsh, R. C. A.: Qualitative dynamics of marine ice sheets, in: Ice in the Climate System, edited by: Peltier, W. R., no. 12 in NATO ASI Series I, 66-99, Springer/Berlin, 1993.

Hindmarsh, R. C. A.: Stability of ice rises and uncoupled marine ice sheets, Ann. Glaciol., 23, 94-104, 1996. 
Hindmarsh, R. C.: An observationally validated theory of viscous flow dynamics at the ice-shelf calving front, J. Glaciol., 58, 375387, doi:10.3189/2012JoG11J206, 2012.

Katz, R. F. and Worster, M. G.: Stability of ice-sheet grounding lines, P. Roy. Soc. A-Math. Phy., 466, 1597-1620, doi:10.1098/rspa.2009.0434, 2010.

MacAyeal, D. R.: Large-scale ice flow over a viscous basal sediment: Theory and Application to Ice Stream B, Antarctica, J. Geophys. Res., 94, 4071-4078, 1989.

MacAyeal, D. R. and Barcilon, V.: Ice-shelf response to ice-stream discharge fluctuations .1. Unconfined ice tongues, J. Glaciol., 34, 121-127, 1988.

Mercer, J. H.: West Antarctic ice sheet and $\mathrm{CO}_{2}$ greenhouse effect: a threat of disaster, Nature, 271, 321-325, doi:10.1038/271321a0, 1978.

Morland, L. W.: Unconfined ice-shelf flow, in: Dynamics of the West Antarctic Ice Sheet, edited by: van derVeen, C. J. and Oerlemans, J., 99-116, Springer, New York, 1987.

Pattyn, F., Schoof, C., Perichon, L., Hindmarsh, R. C. A., Bueler, E., de Fleurian, B., Durand, G., Gagliardini, O., Gladstone, R., Goldberg, D., Gudmundsson, G. H., Huybrechts, P., Lee, V., Nick, F. M., Payne, A. J., Pollard, D., Rybak, O., Saito, F., and Vieli, A.: Results of the Marine Ice Sheet Model Intercomparison Project, MISMIP, The Cryosphere, 6, 573-588, doi:10.5194/tc6-573-2012, 2012.

Pattyn, F., Perichon, L., Durand, G., Favier, L., Gagliardini, O., Hindmarsh, R., Zwinger, T., Albrecht, T., Cornford, S., Docquier, D., Fuerst, J., Goldberg, D., Gudmundsson, G. H., Humbert, A., Huetten, M., Huybrechts, P., Jouvet, G., Kleiner, T., Larour, E., Martin, D., Morlighem, M., Payne, T., Pollard, D., Rueckamp, M., Rybak, O., Seroussi, H., Thoma, M., and Wilkens, N.: Grounding-line migration in plan-view marine ice-sheet models: results of the ice2sea MISMIP3d intercomparison, J. Glaciol., 59, doi:10.3189/2013JoG12J129, accepted, 2013.
Pollard, D. and DeConto, R. M.: Description of a hybrid ice sheetshelf model, and application to Antarctica, Geosci. Model Dev., 5, 1273-1295, doi:10.5194/gmd-5-1273-2012, 2012.

Robison, R. A. V., Huppert, H. E., and Worster, G. M.: Dynamics of viscous grounding lines, J. Fluid Mech., 648, 363-380, doi:10.1017/S0022112009993119, 2010.

Schoof, C.: Ice sheet grounding line dynamics: Steady states, stability, and hysteresis, J. Geophys. Res., 112, F03S28, doi:10.1029/2006JF000664, 2007a.

Schoof, C.: Marine ice-sheet dynamics. Part 1. The case of rapid sliding, J. Fluid Mech., 573, 27-55, doi:10.1017/S0022112006003570, 2007b.

Schoof, C.: Marine ice sheet dynamics, Part 2: A Stokes Flow contact problem, J. Fluid Mech., 679, 122-255, doi:10.1017/jfm.2011.129, 2011.

Schoof, C.: Marine ice sheet stability, J. Fluid Mech., 698, 62-72, doi:10.1017/jfm.2012.43, 2012.

Thomas, R. H. and Bentley, C. R.: A model for Holocene retreat of the West Antarctic Ice Sheet, Quaternary Res., 10, 150-170, doi:10.1016/0033-5894(78)90098-4, 1978.

Weertman, J.: Stability of the junction of an ice sheet and ice shelf, J. Glaciol., 13, 3-11, 1974.

Wilchinsky, A. V.: Studying ice sheet stability using the method of separation of variables, Geophys. Astro. Fluid, 94, 15-45, doi:10.1080/03091920108204130, 2001.

Wilchinsky, A. V.: Linear stability analysis of an ice sheet interacting with the ocean, J. Glaciol., 55, 13-20, 2009. 